\title{
Synthesis Ni-doped CuO nanorods via Successive Ionic Layer Deposition method and their capacitive performance
}

\author{
A. A. Lobinsky, M. V. Kaneva \\ Saint Petersburg State University, Peterhof, 198504 Saint Petersburg, Russia \\ lobinsky.a@gmail.com
}

PACS 81.07.Bc

DOI 10.17586/2220-8054-2020-11-5-608-614

\begin{abstract}
In this work first described the new relatively simple approach to the synthesis of nanolayers of Ni-doped CuO via of Successive Ionic Layer Deposition (SILD) method. The study of Ni-doped CuO nanolayers, synthesized of SILD, has been carried out by HRTEM, XRD, FTIR and XPS spectroscopy methods; it was demonstrated that they had been formed of nanorods with dimensions of about 10-15 nm and tenorite crystal structure $\mathrm{CuO}$ were formed. The research electrochemical properties of nanolayers were carried out in $1 \mathrm{KOH}$ solution by using techniques of cyclic voltammetry and galvanostatic curves method. The electrochemical study of nickel foam electrodes modified by Ni-doped CuO nanolayer prepared by 30 SILD cycles demonstrates that specific capacitance is $154 \mathrm{mAh} / \mathrm{g}(1240 \mathrm{~F} / \mathrm{g})$ at current density $1 \mathrm{~A} / \mathrm{g}$. Repeated cycling after 1000 charge-discharge cycles demonstrates $8 \%$ capacitance fade from the initial value, so such electrodes may be used as effective electroactive materials for alkaline battery and pseudocapacitors.
\end{abstract}

Keywords: copper oxide, nanocrystal, nanorods, successive ionic layer deposition, electrode materials, alkaline battery.

Received: 10 October 2020

\section{Introduction}

As is known, the key role of increase the effectiveness of energy storage devices consist is to create new electrode materials with high energy density and long cycle life [1,2]. From that point of view are of great interest use available oxides and oxyhydroxides of transition metals. These oxides and oxyhydroxides compete with noble metals $\left(\mathrm{RuO}_{2}\right)$, used by electroactive materials in pseudocapacitor. Such materials are environmentally friendly, low cost and at the same time have high specific energy values. Usually, among all oxides and oxyhydroxides, cobalt oxides [3], nickel oxides [4], manganese oxides [5] and iron oxides [6] are used most frequently. However, recent research shows that the most effective electroactive capacity materials should have high intrinsic conductivity and must have a developed morphology of specific shape, which should provide, on the one hand, high specific surface area and, on the other, provide fast diffusion of ions on the surface of the electrode [7].

Recently, copper oxide nanoparticles have been studied as electroactive materials for energy storage devices, as this is one of the oxides with high conductivity and unique morphology [8-11]. In those papers, methods of chemical and eletrochemical deposition from solutions have been used for the synthesis of such nanoparticles. It is worth noticing that previously $\mathrm{CuO}$ nanolayers on the metal surface have been obtained via the SILD method (also called SILAR [12]) to form effective electrodes for supercapacitors. This method is based on the successive and repeated adsorption on the surface of the cations and anions substrate, which interact and give a nanolayer of an insoluble compound. The SILD method has great potential for practical use to form nanolayers on the surface of the products of complex shapes and has been used earlier, for example, for the synthesis of $\mathrm{NiO}_{1+x} \cdot \mathrm{nH}_{2} \mathrm{O}$ [13], $\mathrm{MnOOH}$ [14], $\mathrm{CoOOH}$ [15], $\mathrm{AgMnO}_{2}$ [16], Co-doped $\mathrm{Cu}(\mathrm{OH})_{2}$ [17], $\mathrm{CoO}-\mathrm{NiO}$ solid solution [18], and also Zn-Fe layered double hydroxysulfate $[19,20]$ which can be used as materials for electrocatalytic reforming of ethanol.

In the present paper, we report a new simple route of synthesis of Ni-doped $\mathrm{CuO}$ nanolayers via the SILD method. The obtained nanolayers consist of $\mathrm{CuO}$, including nickel atoms, with nanorods morphology, oriented mainly perpendicular to the substrate surface. We also describe their properties as electroactive materials for the electrode of alkaline batteries and pseudocapacitors.

\section{Experimental}

As a substrate for the synthesis of $\mathrm{CuO}$ nanolayers $0.3 \times 5 \times 25 \mathrm{~mm}$ polycrystalline Ni plates were used, on which electrochemical experiments were performed, and also $0.35 \times 10 \times 25 \mathrm{~mm}$ single-crystal Si plates with $\langle 100\rangle$ orientation, were used for physical characterization. Extra pure water (Milli-Q) was used in all experiments. Si substrates were cleaned in an ultrasonic bath filled with acetone for $10 \mathrm{~min}$. Then plates of Si were sequentially treated for $10 \mathrm{~min}$ in concentrated $\mathrm{HF}$, water, $70 \% \mathrm{HNO}_{3}$, water, $0.1 \mathrm{M} \mathrm{KOH}$ and then flushed out by water. $\mathrm{Ni}$ plates 
were treated according to the technique described in [21] for $15 \mathrm{~min}$ in $6 \mathrm{M} \mathrm{HCl}$ solution, then several times rinsed by water and dried on air at $120^{\circ} \mathrm{C}$ for $30 \mathrm{~min}$.

A solution of mixed $\mathrm{Cu}\left(\mathrm{NH}_{3}\right)_{4}\left(\mathrm{CH}_{3} \mathrm{COO}\right)_{2}$ and $\mathrm{Ni}\left(\mathrm{NH}_{3}\right)_{4}\left(\mathrm{CH}_{3} \mathrm{COO}\right)_{2}$ was prepared by dissolving dry analytical grade salts $\mathrm{Cu}\left(\mathrm{CH}_{3} \mathrm{COO}\right)_{2} \cdot \mathrm{nH}_{2} \mathrm{O}$ and $\mathrm{Ni}\left(\mathrm{CH}_{3} \mathrm{COO}\right)_{2} \cdot \mathrm{nH}_{2} \mathrm{O}(\mathrm{C}=0.01 \mathrm{M})$ and $\mathrm{NH}_{4} \mathrm{CH}_{3} \mathrm{COO}(\mathrm{C}=0.1 \mathrm{M})$ in deionized water. The $\mathrm{pH}$ of the solution was 9.4 and adjusted by the addition of the $\mathrm{NH}_{4} \mathrm{OH}$ solution. The time between the preparation of solutions and synthesis was $0.5 \mathrm{~h}$. For the synthesis of Ni-doped $\mathrm{CuO}$ nanolayers substrate plates were fixed in a holder of special home-made automatic setup and sequentially immersed for $30 \mathrm{~s}$ into a solution of mixed copper and nickel ammonia, then washed from excess reagent in distilled water. On the second step, plates were immersed in a solution of $\mathrm{H}_{2} \mathrm{O}_{2}(3 \%, \mathrm{pH} 9.5$ adjusted by addition of $\mathrm{KOH}$ solution) and again washed in water (Fig. 1). This sequence corresponds to one SILD cycle, which is repeated 30 times to obtain the desired nanolayer thickness. Finished the sample was calcined on argon atmosphere at $200{ }^{\circ} \mathrm{C}$ for 30 minutes at a heating rate of $5{ }^{\circ} \mathrm{C} / \mathrm{min}$.

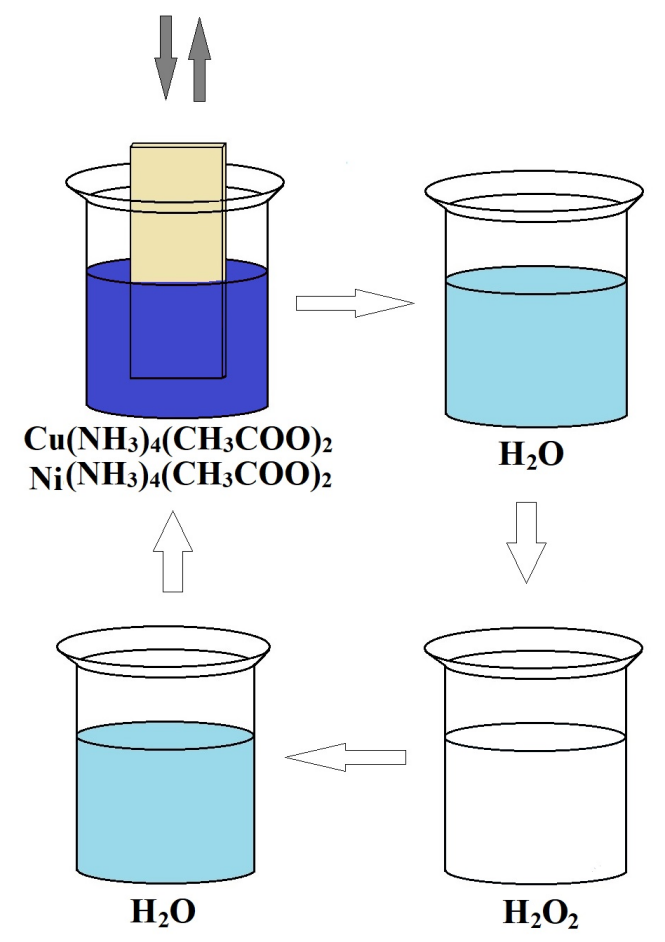

FIG. 1. Scheme of synthesis Ni-doped $\mathrm{CuO}$ nanolayers by SILD method

The obtained samples were characterized by HRTEM, FTIR, XPS and XRD methods. The morphology of synthesized films was investigated by HRTEM (ZeissLibra 200FE, $200 \mathrm{kV}$ ). FTIR transmission spectra of synthesized films on the Si surface were registered by Infraspec FSM 2201 spectrophotometer using a differential technique with respect to spectra of bare silicon plate. XRD patterns were obtained using a Bruker D8 DISCOVER X-ray diffractometer with $\mathrm{CuK} \alpha$ radiation in grazing incidence diffraction geometry $\left(\theta=0.3^{\circ}\right)$. The compositional analyses of the samples were characterized by XPS (ESCALAB 250Xi electron spectrometer, with Al K $\alpha$ radiation $14866 \mathrm{eV}$ ). Hydro-chemical equilibria in solutions were estimated using the simulation program Hydra-Medusa.

The electrochemical measurements of NF electrodes with the synthesized nanolayers were carried out in a threeelectrode electrochemical cell using Elins P-45X potentiostat. The working electrode was prepared by the deposition of $\mathrm{Ni}$-doped $\mathrm{CuO}$ nanolayers on the $\mathrm{Ni}$ foam surface as a result of 30 treatment cycles by the SILD method. Platinum foil serves as the counter electrode and $\mathrm{Ag} / \mathrm{AgCl}$ (aq. $\mathrm{KCl}$ sat.) as the reference electrode. Electrochemical characterization of the films was made by cyclic voltammetry (CV) and galvanostatic charge-discharge (CD) techniques.

The specific capacitance $C(\mathrm{mAh} / \mathrm{g})$ as an electrode for alkaline battery at different current densities can be calculated via eq (1):

$$
C=\int \frac{I d t}{m},
$$

where $I(\mathrm{~mA})$ is a galvanostatic current, $\mathrm{dt}(\mathrm{h})$ is the discharge time of a cycle and $m(\mathrm{~g})$ is the mass of the active material in the film electrode [22]. 
Specific capacitance $C(\mathrm{~F} / \mathrm{g})$ as pseudocapacitor were calculated using the following eq (2):

$$
C=\frac{I \Delta t}{\Delta V m}
$$

where $I(\mathrm{~mA})$ is a galvanostatic current, $\Delta V(\mathrm{mV})$ is the potential window, $\Delta t(\mathrm{~s})$ is the discharge time of a cycle and $m(\mathrm{~g})$ is the mass of the active material in the film electrode [23]. The electroactive mass of Ni-doped $\mathrm{CuO}$ for the working electrode was measured using an OHAUS Pioneer ${ }^{T M}$ PA54C microbalance.

\section{Result and discussion}

The results of the synthesized nanolayers study via the HRTEM method (Fig. 2) demonstrate that nanolayers are formed on the surface after 30 SILD cycles. The nanolayers consist of nanocrystals with nanorods morphology and dimension about $10-15 \mathrm{~nm}$.

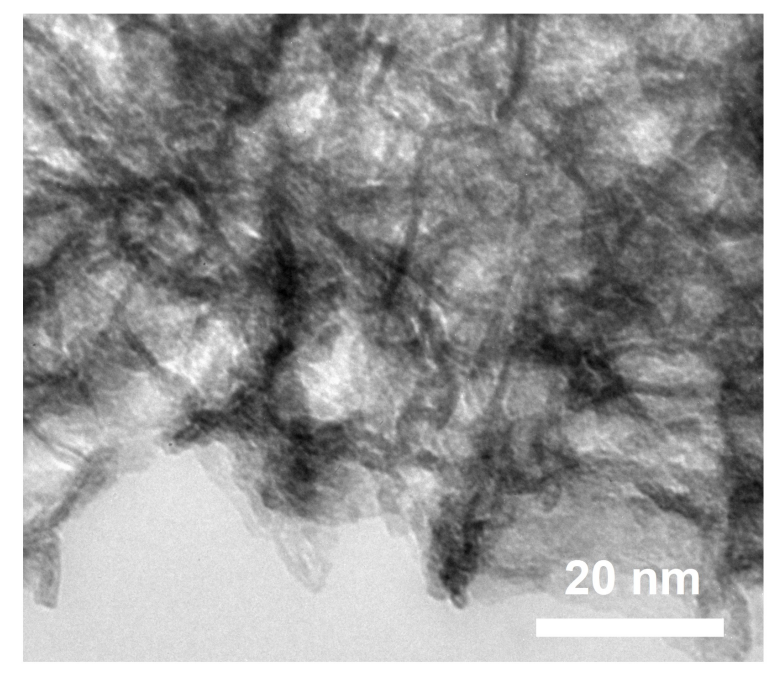

FIG. 2. HRTEM image of copper contained nanolayers

Figure 3 shows the XRD pattern of the synthesized copper contained nanolayers. The latter is in line with crystallographic planes with the orientation of (-111), (111), (-202), (020), (202), (-113) and (311), which are related to a monoclinic crystal lattice of tenorite structure CuO (JCPDS 80-1916) [24].

On the FTIR spectrum (Fig. 4) of the original sample, the broad bands at $3400 \mathrm{~cm}^{-1}$ and $1645 \mathrm{~cm}^{-1}$ are attributed to valence vibrations and deformation vibrations of the hydroxyl groups from $\mathrm{H}_{2} \mathrm{O}$, respectively [25]. The bands with peaks at $1540 \mathrm{~cm}^{-1}$ and $1100 \mathrm{~cm}^{-1}$ correspond to the valence vibrations of the carbonyl group of acetate contained in precursor salt [26]. The bands observed at $530 \mathrm{~cm}^{-1}$ and $450 \mathrm{~cm}^{-1}$ can be corresponding to $\mathrm{Cu}-\mathrm{O}$ vibrations in $\mathrm{CuO}[27]$.

The XPS spectrum shown in Fig. 5 indicates the presence of $\mathrm{Cu}$ and $\mathrm{Ni}$ elements with the atomic ration 1.0/0.38. As shown in Fig. 5(a), two major peaks with binding energy $934.1 \mathrm{eV}$ and $954.1 \mathrm{eV}$ are corresponding to $\mathrm{Cu} 2 \mathrm{p}_{3 / 2}$ and $\mathrm{Cu} 2 \mathrm{p}_{1 / 2}$, indicating that element $\mathrm{Cu}$ is the chemical state of $2+$ in the sample [28]. The Ni $2 \mathrm{p}$ spectra (Fig. 5b) reveal the presence peaks with binding energy $855.4 \mathrm{eV}$ and $872.9 \mathrm{eV}$ are corresponding to $\mathrm{Ni} 2 \mathrm{p}_{3 / 2}$ and $\mathrm{Ni} 2 \mathrm{p}_{1 / 2}$, and also satellites peaks, which indicate of $\mathrm{Ni}$ is the chemical state of 2+ and 3+ [29].

Comparison of research results, conducted via XRD, XPS and FTIR methods, allows us to conclude: obtained nanolayers consist of copper oxide, doped nickel atoms, which are likely to be included in the crystal structure $\mathrm{CuO}$.

We assume that such a structure can possess interesting and practical electrochemical performance, in particular as electrode materials for power sources in the alkaline electrolyte $(1 \mathrm{M} \mathrm{KOH})$. The cyclic voltammograms of the nickel foam (NF) electrode with Ni-doped $\mathrm{CuO}$ nanolayers were recorded in a potential window from 0 to $550 \mathrm{mV}$ vs. $\mathrm{Ag} / \mathrm{AgCl}$ electrode at scanning rates of 5,10,15 and $20 \mathrm{mV} / \mathrm{s}$ (Fig. 6). At a scan rate of $5 \mathrm{mV} / \mathrm{s}$, two redox processes on anodic curve take place in the layer, including the $\mathrm{Cu}^{+} \rightarrow \mathrm{Cu}^{2+}$ transformation at $310 \mathrm{mV}$ and the $\mathrm{Ni}^{2+} \rightarrow \mathrm{Ni}^{3+}$ at $390 \mathrm{mV}$. The proportionality of currents to scan rate provides information that the film is thick enough, and the charge transfer rate is limited by diffusion of charge carriers in the film.

The specific capacitance of the Ni-doped $\mathrm{CuO} \mathrm{NF}$ electrode is calculated from charge-discharge curves (Fig. 7) by eq (1) and eq (2) to be $154 \mathrm{mAh} / \mathrm{g}(1240 \mathrm{~F} / \mathrm{g}), 69 \mathrm{mAh} / \mathrm{g}(1063 \mathrm{~F} / \mathrm{g})$ and $25 \mathrm{mAh} / \mathrm{g}(960 \mathrm{~F} / \mathrm{g})$ at the current densities 


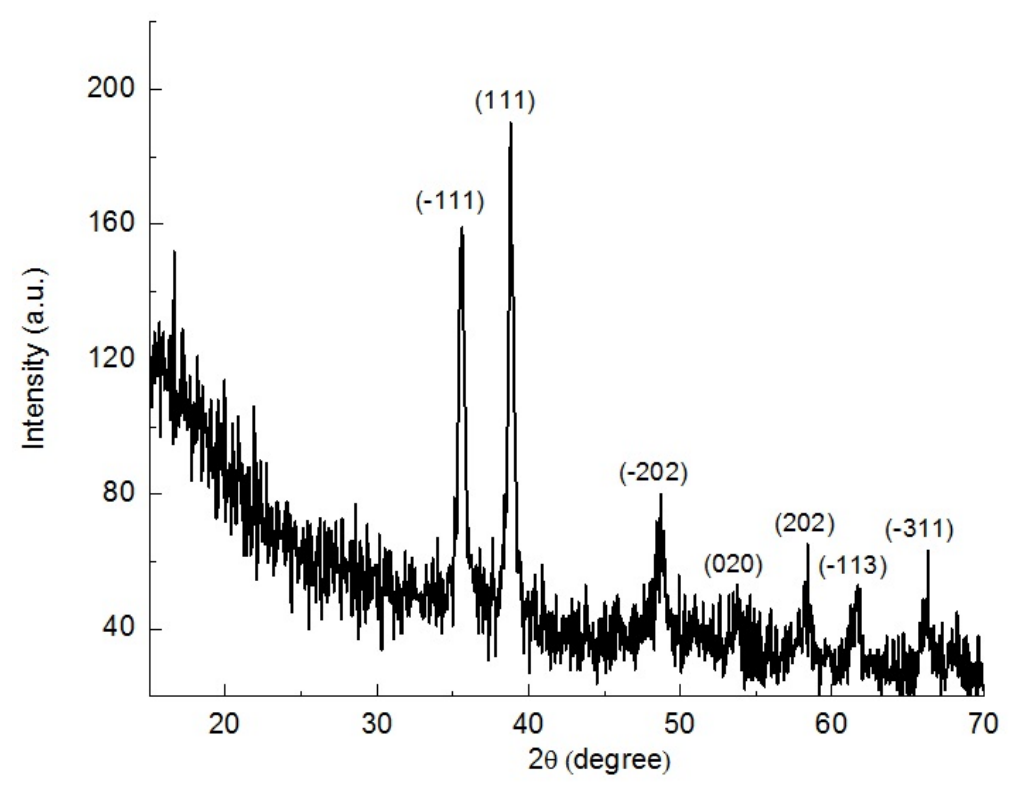

FIG. 3. XRD pattern of copper contained nanolayers

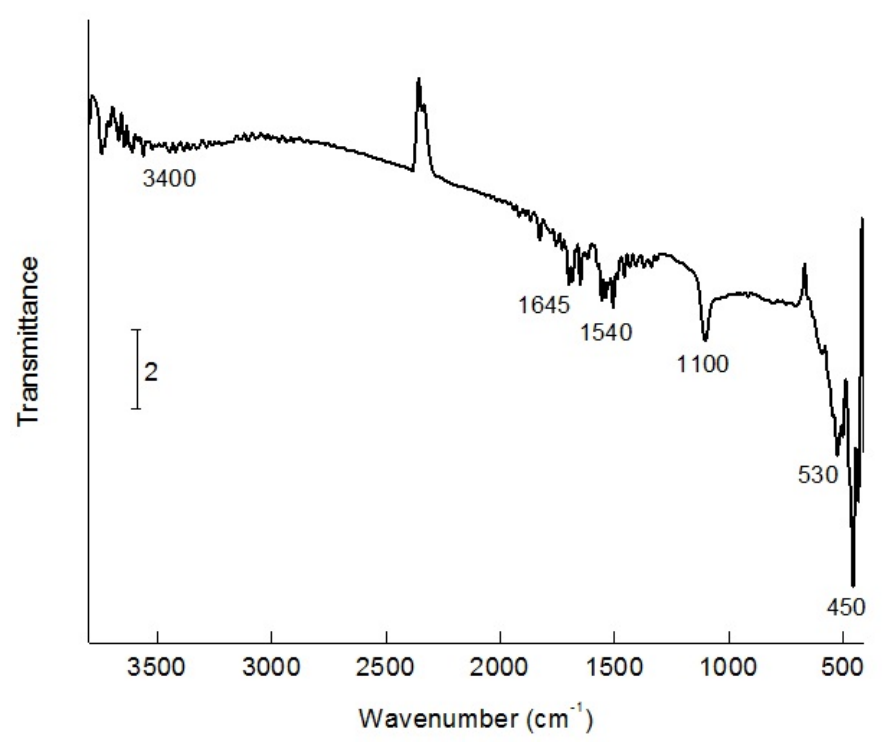

FIG. 4. FTIR transmission spectrum of copper contained nanolayers on silicon

of 1,2 and $5 \mathrm{~A} / \mathrm{g}$, respectively. The high value of specific capacity can be explaining to good conductivity of $\mathrm{CuO}$ and also the significant contribution of nickel atoms in pseudocapacity for this sample.

Cyclic stability is also an important property for electroactive materials. The capacity retention of the NF electrode with $\mathrm{Ni}$-doped $\mathrm{CuO}$ nanolayers after 1000 charge-discharge cycles at current density $2 \mathrm{~A} / \mathrm{g}$ was kept $92 \%$ of its initial capacity (Fig. 8) that shows good cycling stability of this material. High cycling stability can be explained by the feature morphology of ultrathin nanocrystals of $\mathrm{CuO}$ which provide fast diffusion of ions on the electrode surface and while not being destroyed in charge-discharge process.

We believe that these electrochemical capacity characteristics for electrodes of alkaline battery and pseudocapacitor based on Ni-doped $\mathrm{CuO}$ nanorods, synthesized via the SILD method, can be improved using a new scheme of their synthesis, including nanocomposite with carbon materials (CNT, graphene), the formation of which has been obtained after a new sequence of reagent treatment. However, these experiments fall outside the scope of the present paper. Undoubtedly, the synthesized nanolayers can also form a basis for effective electrodes of electrocatalysts and electrochemical sensors, etc. 


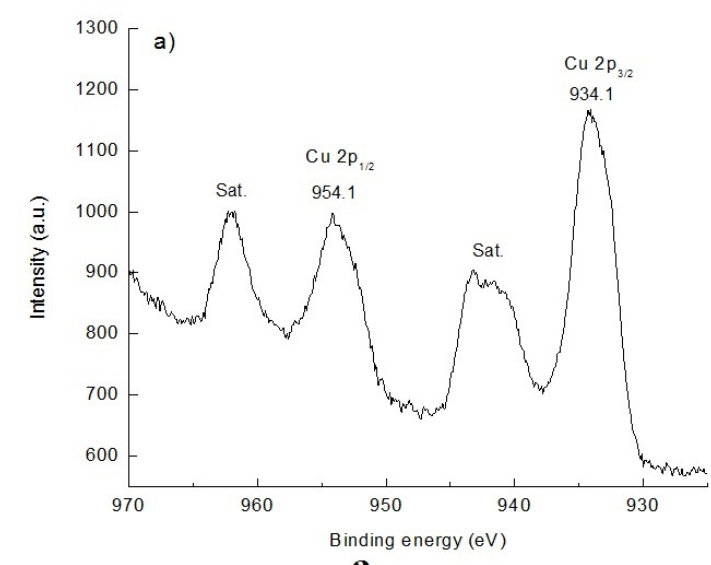

a

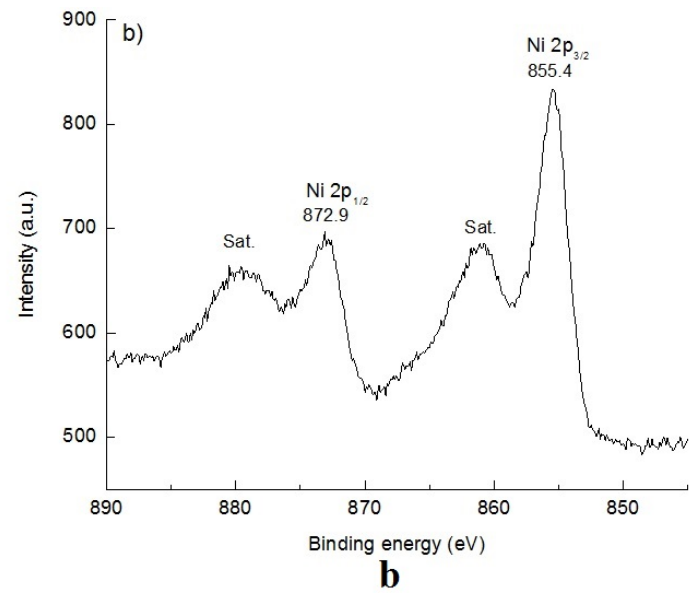

b

FIG. 5. XPS spectra Cu2p (a) and Ni2p (b) of copper contained nanolayers on the silicon surface

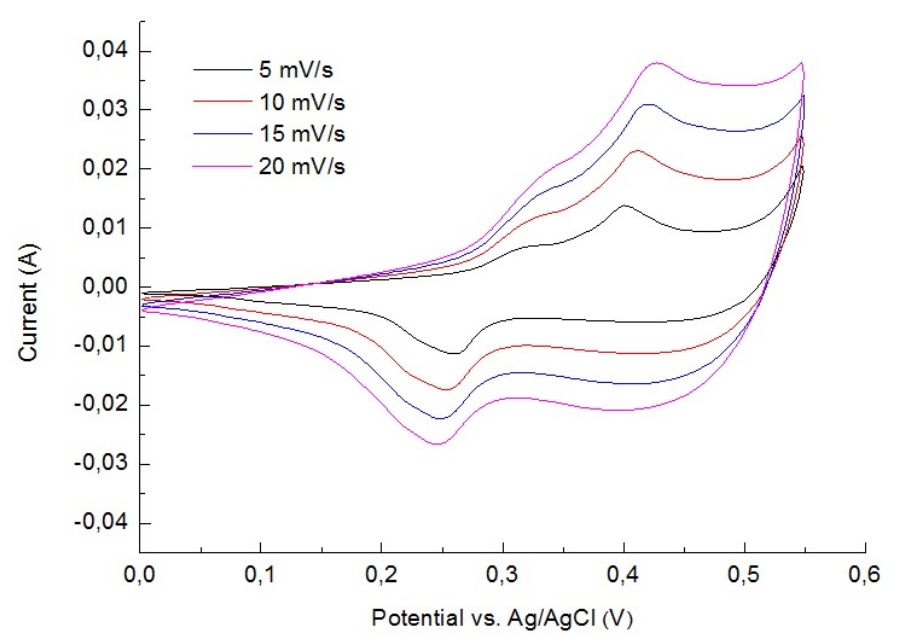

FIG. 6. CVA curves for NF electrode with Ni-doped $\mathrm{CuO}$ nanolayers at a scan rate of 5, 10, 15 and $20 \mathrm{mV} / \mathrm{s}$

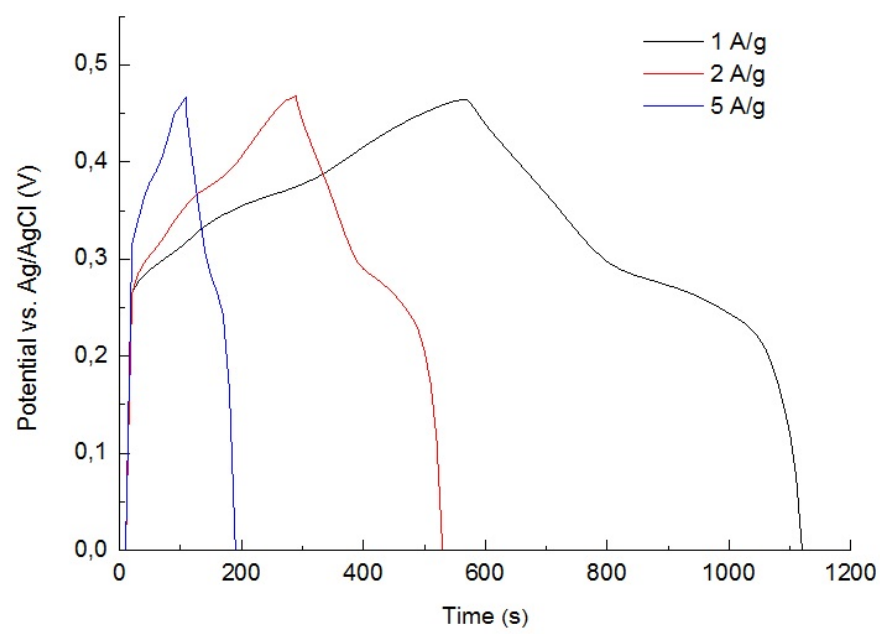

FIG. 7. Galvanostatic charge-discharge curves of the electrode with Ni-doped $\mathrm{CuO}$ nanolayers 


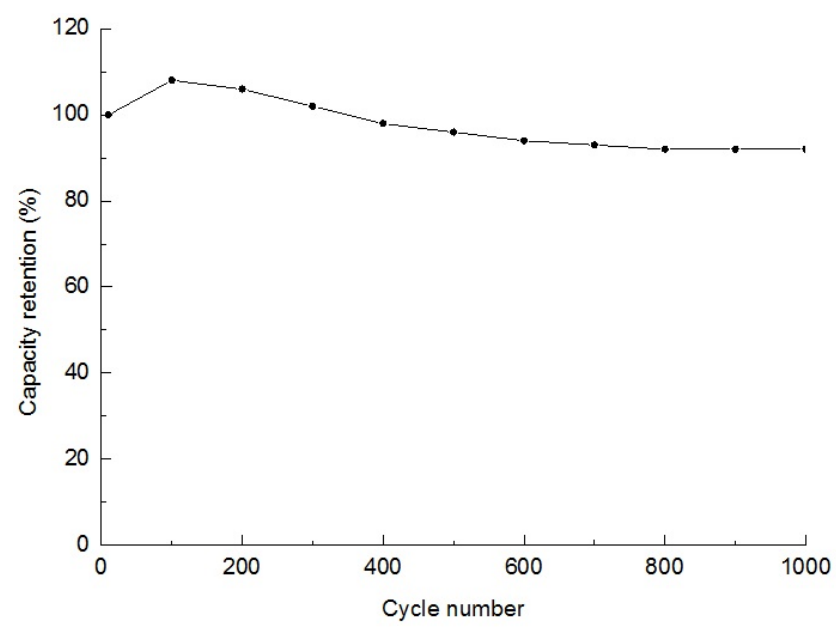

FIG. 8. The cycling stability for the NF electrode with Ni-doped $\mathrm{CuO}$ nanolayers at $2 \mathrm{~A} / \mathrm{g}$

\section{Conclusion}

In summary, the possibility of obtaining $\mathrm{Ni}$-doped $\mathrm{CuO}$ nanolayers via the SILD method using mixed copper and nickel ammonia aqueous solution and hydrogen peroxide solution was shown. The results show the synthesized nanolayers were formed nanocrystals of Ni-doped $\mathrm{CuO}$ the thickness of about 10-15 nm with tnanorod morphology and the monoclinic tenorite crystal structure of $\mathrm{CuO}$. The electrochemical study of Ni-doped CuO nanolayersmodified nickel foam electrodes, prepared by 30 SLID cycles, demonstrates that the specific capacitance is $154 \mathrm{mAh} / \mathrm{g}$ $(1240 \mathrm{~F} / \mathrm{g})$ at a current density of $1 \mathrm{~A} / \mathrm{g}$. Repeated cycling for 1000 charge-discharge cycles demonstrates a relatively small $8 \%$ capacitance fade. The electrode based on $\mathrm{Ni}$-doped $\mathrm{CuO}$ nanolayers had shown high energy density and the long-term electrochemical cycling stability. Thus this material can be a potential application as electroactive materials for alkaline battery and pseudocapacitors.

\section{Acknowledgments}

This research was financial supported by the Grant of President of Russian Federation MK-2860.2019.3. The authors are grateful to the Centers for X-ray diffraction studies and Nanotechnology of Saint-Petersburg State University.

\section{References}

[1] Zhong C., Deng Y., Hu W., Qiao J., Zhang L., Zhang J. A review of electrolyte materials and compositions for electrochemical supercapacitors. Chem. Soc. Rev., 2015, 44, P. 7484-7539.

[2] Wang F., Wu X., Yuan X. et al. Latest advances in supercapacitors: from new electrode materials to novel device designs. Chem. Soc. Rev., 2017, 46, P. 6816-6854.

[3] Meher S.K., Rao G.R. Ultralayered Co3O4 for high-performance supercapacitor applications. J. Phyl. Chem. C, 2011,115, P. $15646-15654$.

[4] Zhiyi Zhang, Qiuyue Gao, Haibo Gao, Zhenyu Shi, Junwei Wu, Mingjia Zhi, Zhanglian Hong. Nickel oxide aerogel for high-performance supercapacitor electrode. RSC Adv., 2016, 6, P. 112620-112624.

[5] Zhu G., He Z., Chen J. et al. Highly conductive threedimensional $\mathrm{MnO}_{2}$-carbon nanotube-graphene-Ni hybrid foam as a binder-free supercapacitor electrode. Nanoscale, 2014, 6, P. 1079-1085.

[6] Yang P., Ding Y., Lin Z. et al. Low-cost high-performance solid-state asymmetric supercapacitors based on $\mathrm{MnO}_{2}$ nanowires and Fe $\mathrm{O}_{3}$ nanotubes. Nano Lett., 2014, 14, P. 731-736.

[7] Choi C, Ashby D.S., Butts D.M. et al. Achieving high energy density and high power density with pseudocapacitive materials. Nat. Rev. Mater, 2020, 5, P. 5-19.

[8] Liu Y., Cao X., Jiang D., Jia D., Liu J. Hierarchical CuO nanorod arrays in situ generated on three-dimensional copper foam via cyclic voltammetry oxidation for high-performance supercapacitors. J. Mater. Chem. A, 2018, 6, P. 10474-10483.

[9] Deepak P. Dubal, Girish S. Gund, Chandrakant D. Lokhande, Rudolf Holze. CuO cauliflowers for supercapacitor application: Novel potentiodynamic deposition. Materials Research Bulletin, 2013, 48, P. 923-928.

[10] Mohammad Bagher Gholivand, HamidHeydari, Abbas Abdolmaleki, Hamid Hosseini. Nanostructured CuO/PANI composite as supercapacitor electrode material. Materials Science in Semiconductor Processing, 2015, 30, P. 157-161.

[11] Seyyed E Moosavifard, Maher F El-Kady, Mohammad S Rahmanifar, Richard B Kaner, Mir F Mousavi. Designing 3D highly ordered nanoporous $\mathrm{CuO}$ electrodes for high-performance asymmetric supercapacitors. ACS Appl. Mater. Interfaces, 2015, 7(8), P. 4851-60.

[12] Tolstoy V.P., Kodintsev I.A., Reshanova K.S., Lobinsky A.A. A brief review of metal oxide (hydroxide)-graphene nanocomposites synthesis by layer-by-layer deposition from solutions and synthesis. Reviews on advanced materials science, 2017, 49(1), P. $28-37$.

[13] Lobinsky A.A., Tolstoy V.P., Gulina L.B. A novel oxidation-reduction route for successive ionic layer deposition of $\mathrm{NiO}_{1+x} \cdot \mathrm{nH}_{2} \mathrm{O}$ nanolayers and their capacitive performance. Materials Research Bulletin, 2016, 76, P. 229-234. 
[14] Lobinsky A.A., Tolstoy V.P. Synthesis of $\gamma-\mathrm{MnOOH}$ nanorods by successive ionic layer deposition method and their capacitive performance. Journal of Energy Chemistry, 2017, 26, P. 336-339.

[15] Lobinsky A.A., Tolstoy V.P. Red-ox reactions in aqueous solutions of $\mathrm{Co}(\mathrm{OAc})_{2}$ and $\mathrm{K}_{2} \mathrm{~S}_{2} \mathrm{O}_{8}$ and synthesis of CoOOH nanolayers by the SILD method. Nanosystems: Physics, Chemistry, Mathematics, 2015, 6(6), P. 843-849.

[16] Kodintsev I.A., Tolstoy V.P. Lobinsky A.A. Room temperature synthesis of composite nanolayer consisting of $\mathrm{AgMnO}_{2}$ delafossite nanosheets and Ag nanoparticles by successive ionic layer deposition and their electrochemical properties. Materials Letters, 2017, 196, P. 54-56.

[17] Kodintsev I.A., Martinson K.D., Lobinsky A.A., Popkov V.I. Successive ionic layer deposition of Co-doped Cu(OH) 2 nanorods as electrode material for electrocatalytic reforming of ethanol. Nanosystems: Physics, Chemistry, Mathematics, 2019, 10(5), P. 573-578.

[18] Kodintsev I.A., Martinson K.D., Lobinsky A.A., Popkov V.I. SILD synthesis of the efficient and stable electrocatalyst based on CoO-NiO solid solution toward hydrogen production. Nanosystems: Physics, Chemistry, Mathematics, 2019, 10(6), P. 681-685.

[19] Dmitriev D.S., Popkov V.I., Layer by layer synthesis of zinc-iron layered hydroxy sulfate for electrocatalytic hydrogen evolution from ethanol in alkali media. Nanosystems: Physics, Chemistry, Mathematics, 2019, 10(4), P. 480-487.

[20] Popkov V.I., Tolstoy V.P., Semenov V.G., Synthesis of phase-pure superparamagnetic nanoparticles of $\mathrm{ZnFe}_{2} \mathrm{O}_{4}$ via thermal decomposition of zinc-iron layered double hydroxysulphate. Journal of Alloys and Compounds, 2020, 813, P. 152179.

[21] Tolstoy V.P., Lobinsky A.A., Levin O.V., Kuklo L.I. Direct synthesis of $\mathrm{Ni}_{2} \mathrm{Al}(\mathrm{OH})_{7-x}\left(\mathrm{NO}_{3}\right)_{x} \cdot \mathrm{nH}_{2} \mathrm{O}$ layered double hydroxide nanolayers by SILD and their capacitive performance. Materials Letters, 2015, 139, P. 4-6.

[22] Tolstoy V.P., Lobinsky A.A. Synthesis of 2D Zn-Co LDH nanosheets by a successive ionic layer deposition method as a material for electrodes of high-performance alkaline battery-supercapacitor hybrid devices. RSC Advances, 2018, 8, P. 29607-29612.

[23] Liu Y., Cao X., Jiang D., Jia D., Liu J. Hierarchical CuO nanorod arrays in situ generated on three-dimensional copper foam via cyclic voltammetry oxidation for high-performance supercapacitors. J. Mater. Chem. A, 2018, 6, P. 10474-10483.

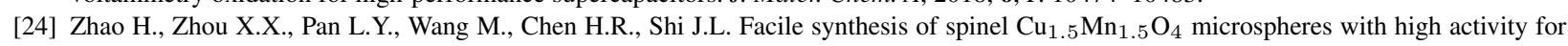
the catalytic combustion of diesel soot. RSC Adv., 2017, 7, P. 20451-20459.

[25] Xie J., Cao H., Jiang H., Chen Y., Shi W., Zheng H., Huang Y. $\mathrm{Co}_{3} \mathrm{O}_{4}$-reduced graphene oxide nanocomposite as an effective peroxidase mimetic and its application in visual biosensing of glucose. Anal. Chim. Acta, 2013, 796, P. 92.

[26] Zhangpeng Li, Jinqing Wang, Lengyuan Niu, Jinfeng Sun, Peiwei Gong, Wei Hong, Limin Ma, Shen-grong Yang Rapid. Synthesis of graphene/cobalt hydroxide composite with enhanced electrochemical performance for supercapacitors. Journal of Power Sources, 2014, 245, P. 224-231.

[27] Ethiraj A.S., Kang D.J., Synthesis and characterization of $\mathrm{CuO}$ nanowires by a simple wet chemical method. Nanoscale Research Letters, 2012, 7(1), P. 70.

[28] Xu L., Zhang H., Li J. at all. Designing core-shell Ni(OH) 2 @ CuO nanowire arrays on 3D copper foams for high-performance asymmetric supercapacitors. Chem. Electro Chem., 2019, 6, P. 5462-5468.

[29] Gui Chen, Lingjing Chen, Siu-Mui Ng, Tai-Chu Lau. Efficient chemical and visible-light-driven water oxidation using nickel complexes and salts as precatalysts. Chem. Sus. Chem., 2014, 7, P. 127-134. 